\title{
Numerical study of critical properties and hidden orders in dimerized spin ladders
}

\author{
Sandra J. Gibson, ${ }^{1}$ R. Meyer, ${ }^{1,2}$ and Gennady Y. Chitov ${ }^{1}$ \\ ${ }^{1}$ Department of Physics, Laurentian University, Sudbury, Ontario P3E 2C6, Canada \\ ${ }^{2}$ Department of Mathematics and Computer Science, Laurentian University, Sudbury, Ontario P3E 2C6, Canada
}

(Received 29 September 2010; revised manuscript received 18 January 2011; published 29 March 2011)

\begin{abstract}
Dimerized antiferromagnetic spin- $\frac{1}{2}$ ladders are known to exhibit a quantum critical phase transition in the ground state, the existence or absence of which is dependent on the dimerization pattern of the ladder. The gapped phases cannot be distinguished by the conventional Landau long-range order parameter. However, they possess a nonlocal (hidden) string-order parameter, which is nonzero in one phase and vanishes in the other. We use an exact diagonalization technique to calculate ground-state energies, energy gaps, and string-order parameters of dimerized two- and three-leg Heisenberg ladders, as well as a critical scaling analysis to yield estimates of the critical exponents $v$ and $\beta$.
\end{abstract}

DOI: 10.1103/PhysRevB.83.104423

PACS number(s): 75.10.Kt, 75.10.Pq, 75.10.Dg

\section{INTRODUCTION}

Spin ladders have been the focus of significant theoretical interest in the past decade or longer. This work has been grounded by the experimental discovery of several spin-ladder and spin-Peierls compounds exhibiting various fascinating phenomena. ${ }^{1}$ Many of the important features of these materials have long been understood (For reviews see, e.g., Refs. 1,2). However, significant discoveries may yet be made as crucial questions about the underlying nature of these systems remain.

One of the most peculiar properties of spin ladders is that the existence of a gap (i.e., mass) depends on the number of legs. The spin excitations in a $m$-leg spin ladder are gapped if $m$ is even, and the system is gapless (quantum critical) when the number of legs $m$ is odd. The even- $m$-leg ladders are interesting examples of spin liquids, which are currently under enormous scrutiny. The spin liquid has, by definition, a gap that is not due to long-range order or apparent symmetry breaking. This is hard to understand in terms of the basic postulates of the conventional Landau paradigm for phase transitions. ${ }^{3}$

The purpose of this study is to explore the question of the existence of "hidden orders" associated with the massive quantum phases of dimerized Heisenberg ladders with antiferromagnetic couplings. It is known that single dimerized Heisenberg spin- $\frac{1}{2}$ chains are gapped, and that when two chains are coupled into a ladder, the system is gapped even without dimerization. ${ }^{2}$ Counterintuitively, the dimerized twoand three-leg ladders can also be gapless. The critical (gapless) lines of the dimerized two- and three-leg ladders were first conjectured in Ref. 4 and have been confirmed by subsequent numerical and analytical work. ${ }^{5-12}$ The characterization of the unconventional quantum phases in such systems in terms of more exotic types of orders may ultimately help to uncover novel physics that lies beyond the Landau paradigm.

We present numerical results on finite-size spin- $\frac{1}{2}$ systems with total number of spins $(N)$ of up to $N=30$. These results are obtained here using an exact diagonalization (ED) technique based on the Lanczos algorithm. ${ }^{13}$ The total available computer memory is the most important factor limiting the maximum system sizes accessible using this method. This is because the dimension of the Hamiltonian matrix grows exponentially fast (as $2^{N}$ for a spin- $\frac{1}{2}$ state). Although restricted to relatively small system sizes, the ED method is considered an "unbiased method" useful for calculating almost any physical quantity. Our implementation of the Lanczos algorithm uses periodic boundary conditions and includes an explicit restart step with more than five restarts to mitigate loss of orthogonality in the Lanczos basis.

\section{TWO-LEG LADDER}

We begin by presenting the results for a two-legged ladder with intrinsic dimerization. A similar program is carried out for the three-leg ladder, detailed in Sec. III. In line with the previous work, ${ }^{2,14}$ which used the bond-mean-field theory, ${ }^{15}$ we have considered the two possible dimerization patterns of the ladder (staggered and columnar) on an equal footing (see Fig. 1).

The Hamiltonian of the dimerized spin ladder with $m$ legs is given by

$$
\begin{aligned}
H= & \sum_{n=1}^{N} \sum_{\alpha=1}^{m} J_{\alpha}(n) \mathbf{S}_{\alpha}(n) \cdot \mathbf{S}_{\alpha}(n+1) \\
& +J_{\perp} \sum_{n=1}^{N} \sum_{\alpha=1}^{m-1} \mathbf{S}_{\alpha}(n) \cdot \mathbf{S}_{\alpha+1}(n),
\end{aligned}
$$

where the dimerization occurs along the chains $(\alpha=1, \ldots, m)$ only, with the rung coupling $J_{\perp}$ taken as constant. All the spin exchange couplings are antiferromagnetic. The dimerization patterns are then defined as

$$
\begin{array}{cc}
J_{\alpha}(n)=J\left[1+(-1)^{n+\alpha} \delta\right] \quad(\text { staggered }), \\
J_{\alpha}(n)=J\left[1+(-1)^{n} \delta\right] \quad \text { (columnar), }
\end{array}
$$

with periodic boundary conditions along the chains and open boundary conditions in the rung directions.

\section{A. Ground-state energies and gaps}

We find that the ladders with the columnar dimerization order always have the lower ground-state energy, and that they are always gapped. The results for the ground-state energies of the ladders with $N=24$ are shown in Fig. 2 are very close 


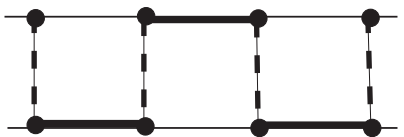

(a)

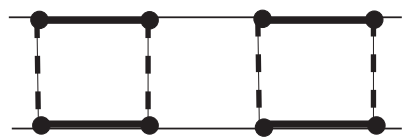

(b)
FIG. 1. Dimerized two-leg ladder. Bold, thin, and dashed lines represent the stronger or weaker chain coupling $J(1 \pm \delta)$ and rung coupling $J_{\perp}$, respectively. Dimerization patterns: (a) staggered; (b) columnar.

to earlier predictions for these systems from the mean-field theory. ${ }^{12}$

Also in agreement with the earlier mean-field predictions, ${ }^{12}$ our ED calculations confirm that the existence or absence of the quantum critical phase transition between gapped phases depends on the dimerization pattern. The columnar arrangement of the strong and weak couplings on the legs is never critical, whereas the staggered configuration possesses a quantum critical point. The characteristic results for the singlet-triplet gaps are shown in Fig. 3.

To take into account the finite-size effects in the gaps obtained by ED and use these data to extract the critical indices near the transition point, we have applied a finite-size scaling analysis. ${ }^{16}$ If we denote the critical coupling where the gap vanishes as $J_{\perp c}$ (the phase diagram of the dimerized two-leg ladder is shown in Fig. 4) and the reduced coupling as $g \equiv \frac{J_{\perp}-J_{\perp c}}{J_{\perp c}}$, the gap $\Delta_{L}(g)$ in the system of the linear dimension $L$ can be expressed as

$$
\Delta_{L}(g)=L^{-1} f\left(g L^{\frac{1}{v}}\right)
$$

where $f\left(g L^{\frac{1}{v}}\right)$ is a dimensionless scaling function and $v$ is the critical index of the correlation length. The unknown scaling function $f$ is determined by the best collapse of the finite-size ED data. The fitting gives $v \approx 0.75$ (see Fig. 5).

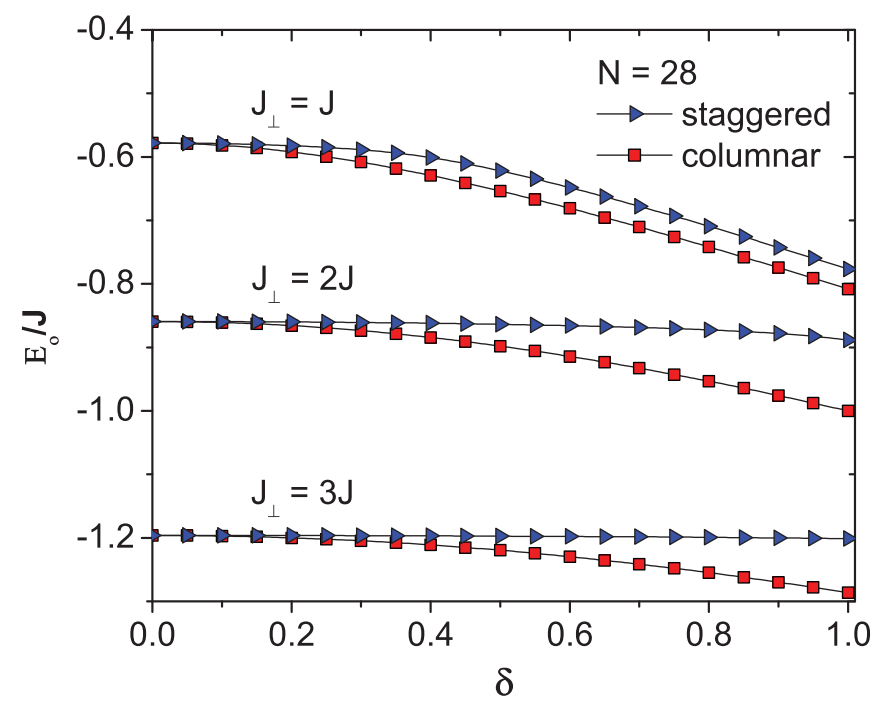

FIG. 2. (Color online) The ground-state energies for the staggered and columnar two-leg ladder calculated by exact diagonalization for $N=28$.

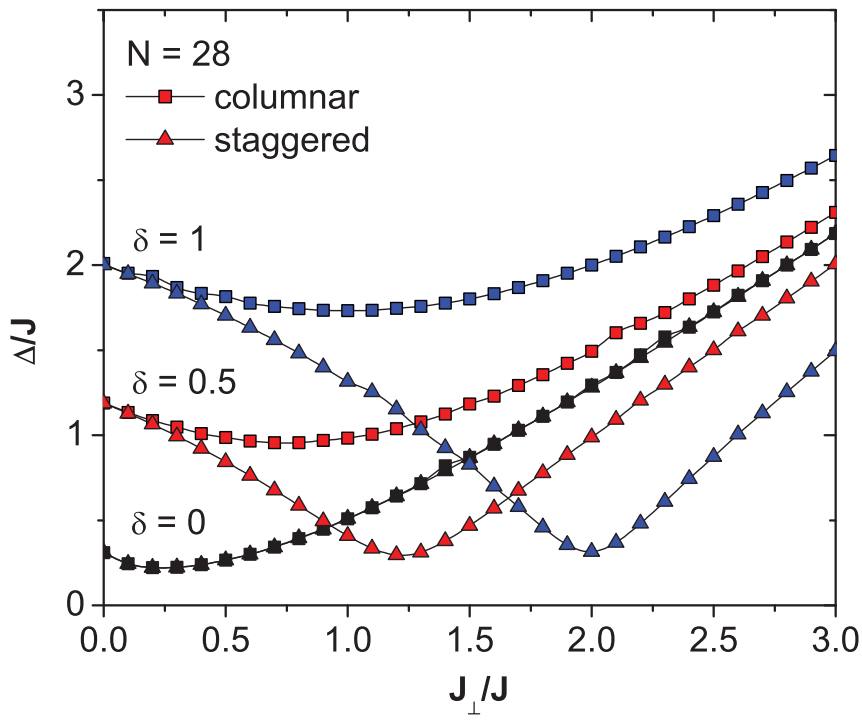

FIG. 3. (Color online) The ground-state energy gap for the staggered and columnar two-leg ladder calculated by exact diagonalization for $N=28$.

\section{B. String-order parameter}

In the absence of the conventional Landau order parameter, the different gapped phases in the ladder can be distinguished only by a nonlocal string-order parameter (SOP), which is nonzero in one phase and vanishes in the other. The SOP was first introduced in 1989 by den Nijs and Rommelse, ${ }^{17}$ who related it to the gapped or Haldane phase in the spin-1 Heisenberg chain. In that system there is no local symmetry breaking, and therefore no local-order parameter can be identified. However, the SOP detects the hidden $Z_{2} \otimes Z_{2}$

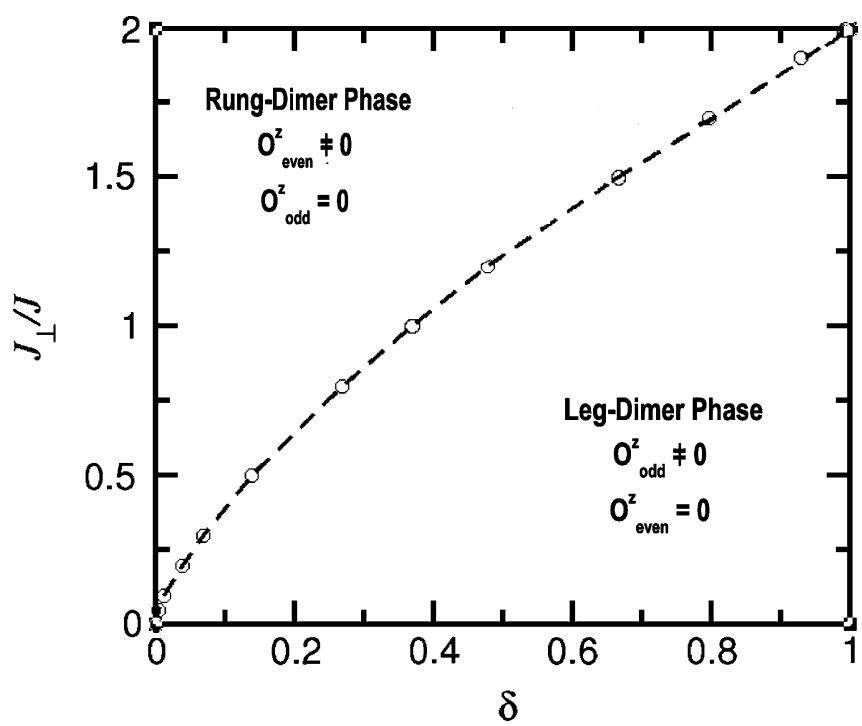

FIG. 4. Two-leg ladder; critical line $J_{\perp c}(\delta)$ where the gap of the staggered phase vanishes. Adapted from Ref. 12, original data from Ref. 9. 

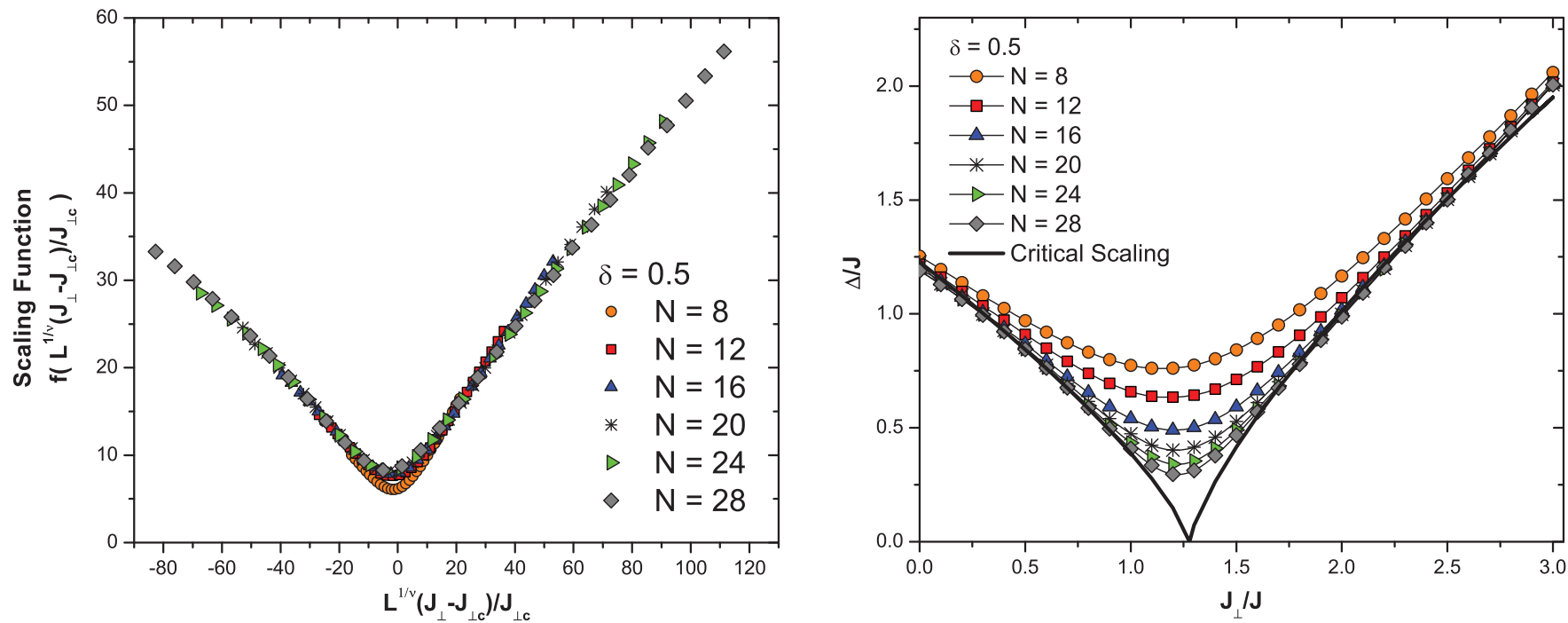

FIG. 5. (Color online) The collapsed scaling function (left-hand panel) and gap (right-hand panel) for the staggered two-leg ladder with $\delta=0.5$, calculated for $N=8$ through 28 along with the fit using optimized parameters $v \approx 0.755$ and $J_{\perp c} \approx 1.27$.

symmetry breaking ${ }^{18}$ in the Haldane phase. The SOP notion was later generalized for two-leg spin ladders as ${ }^{19-25}$

$$
\begin{aligned}
O_{\text {odd/even }}^{z} \equiv & -\lim _{|n-m| \rightarrow \infty}\left\langle S_{\text {rung/diag }}^{z}(n)\right. \\
& \left.\times \exp \left[i \pi \sum_{l=n+1}^{m-1} S_{\text {rung } / \text { diag }}^{z}(l)\right] S_{\text {rung } / \text { diag }}^{z}(m)\right\rangle
\end{aligned}
$$

where $\quad S_{\text {rung }}^{z}(n) \equiv S_{1}^{z}(n)+S_{2}^{z}(n) \quad$ and $\quad S_{\text {diag }}^{z}(n) \equiv S_{1}^{z}(n)+$ $S_{2}^{z}(n+1)$. The even and odd SOPs have been shown to be mutually exclusive in several numerical and analytical studies. ${ }^{11,23}$ Previous numerical studies ${ }^{11,19}$ have assumed a staggered dimerization, while here the SOPs were calculated

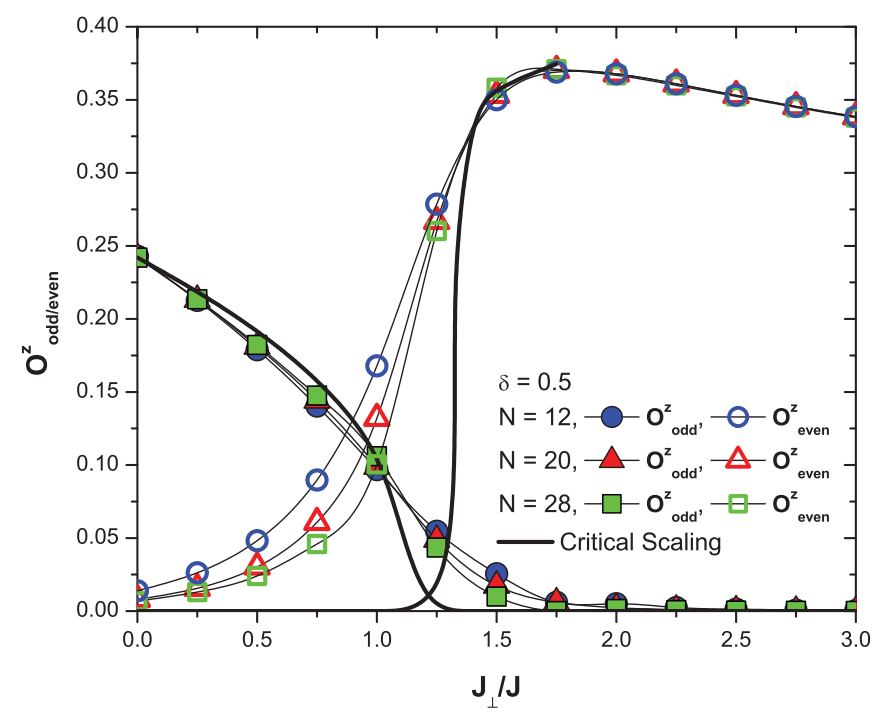

FIG. 6. (Color online) The odd and even SOPs of the staggered two-leg ladder with $\delta=0.5$. The ED data for ladders with number of spins $N=12$ to 28 are shown, along with the critical scaling curves. for both configurations by the ED method. In agreement with the previous studies, we find that the SOPs are featureless for the columnar phase: In the whole phase space domain $\left(J_{\perp}, \delta\right)$ the even SOP is nonzero, while the odd SOP vanishes.

We find for the staggered phase that the even or odd SOP is nonzero only in the rung-dimer and leg-dimer phases, respectively, and vanishes at the critical point. The calculated SOPs are shown in Fig. 6. The SOP vanishes at the critical point as $O_{\sharp}^{z} \propto g^{2 \beta}$ where $\beta$ is the critical index of the order parameter. $^{20,26}$ Then the SOP in the system of length $L$ scales as

$$
O_{L}^{z}(g)=L^{-\frac{2 \beta}{v}} \tilde{f}\left(g L^{\frac{1}{v}}\right)
$$

where the scaling function $\tilde{f}$ is determined as for the gap by the requirement to collapse the numerical data for the systems of different sizes onto the same curve. From the analysis of the ED data for $\delta=0.5$ we obtain a critical index $\beta_{\text {odd }} \approx 0.25$ for the odd string order and $\beta_{\text {even }} \approx 0.025$ for the even string order. The collapse of the scaling functions is shown for $\delta=0.5$ in Fig. 7. The same critical scaling analysis was performed for several values of $\delta$, and these results are tabulated in Table I.

TABLE I. Critical scaling indices of the two-leg ladder.

\begin{tabular}{lclll}
\hline \hline$\delta$ & Even or odd & \multicolumn{1}{c}{$\beta$} & \multicolumn{1}{c}{$v$} & $J_{\perp c} / J$ \\
\hline 0.25 & Even & 0.05294 & 0.758 & 0.775 \\
0.25 & Odd & 0.285 & 0.787 & 0.759 \\
0.5 & Even & 0.0251 & 0.726 & 1.32 \\
0.5 & Odd & 0.215 & 0.698 & 1.21 \\
0.75 & Even & 0.0687 & 0.764 & 1.62 \\
0.75 & Odd & 0.235 & 0.648 & 1.64 \\
1.0 & Even & 0.0124 & 0.7306 & 2.08 \\
1.0 & Odd & 0.227 & 0.7013 & 1.98 \\
\hline \hline
\end{tabular}



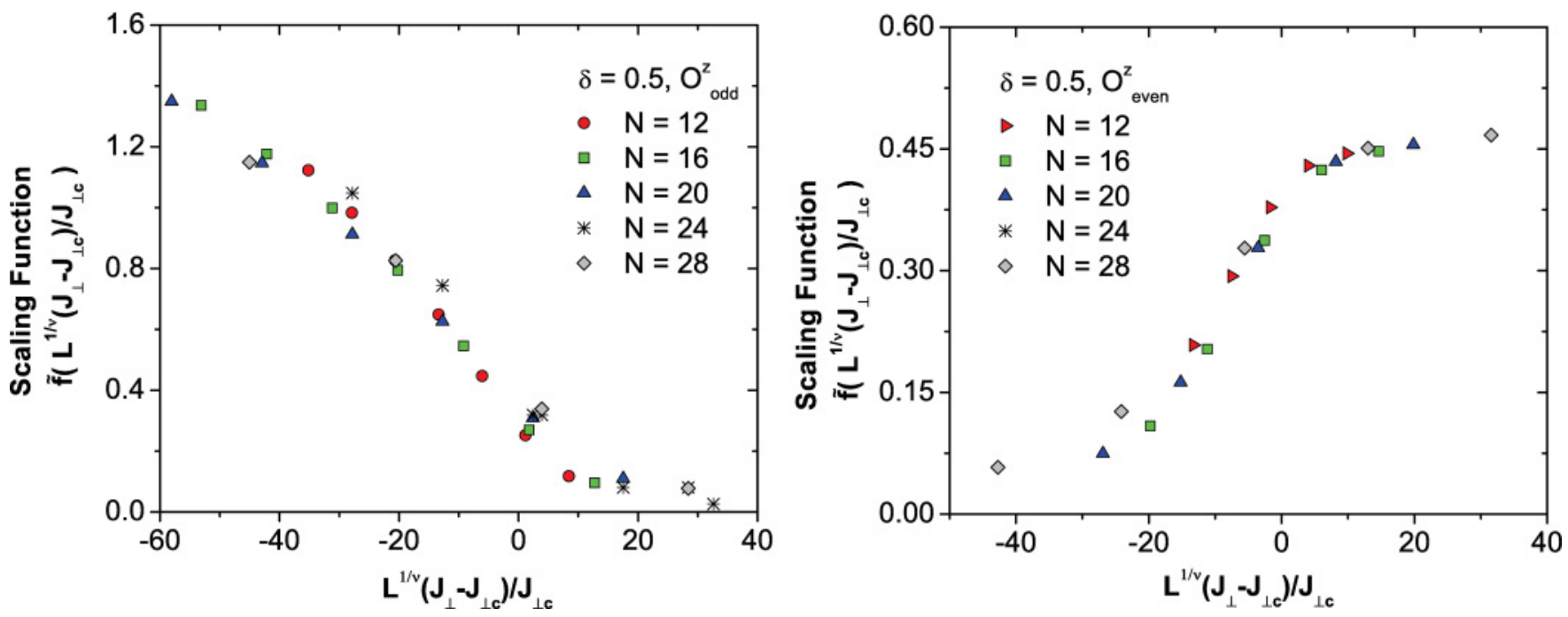

FIG. 7. (Color online) The left-hand (right-hand) panel shows the fitted scaling function for the odd (even) SOP of the staggered two-leg ladder with $\delta=0.5$. The data collapse for the optimized scaling parameters $v=0.7, J_{\perp c}=1.21$, and $\beta_{\text {odd }}=0.25\left(v=0.74, J_{\perp c}=1.32\right.$, and $\left.\beta_{\text {even }}=0.025\right)$.

\section{THREE-LEG LADDER}

Similar to the two-legged case the dimerized three-leg ladder occurs in two distinct configurations, the staggered and columnar patterns (see Fig. 8). The Hamiltonian of the intrinsically dimerized three-leg ladder is given by Eq. (1) with $m=3$ and like the two-legged ladder the staggered or columnar patterns are given by the coupling $J_{\alpha}(n)$ defined by Eqs. (2) and (3), respectively.

\section{A. Ground-state energies and gaps}

The ground-state energies were calculated by the ED method for the three-legged ladder with $N=24$ total number of spins. The columnar pattern was found to have the consistently lower energies throughout the parameter space, in agreement with the previous results from the bond-mean-field theory. ${ }^{12}$ The ED results for the energy gaps, given in Fig. 9 for $N=24$, show that the columnar configuration is always gapped, and the staggered pattern approaches gaplessness at a critical value of rung coupling $J_{\perp c}$. Similar to the two-legged case, the appearance of criticality in the three-legged ladder depends on the dimerization pattern. The phase diagram of the staggered ladder is given in Fig. 10. The ground states of these ladders have been found to be well described by short-range

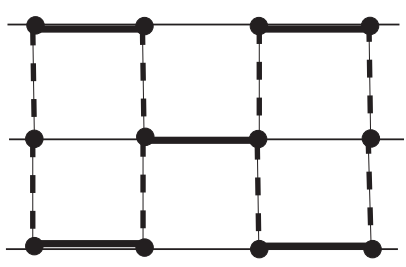

(a)

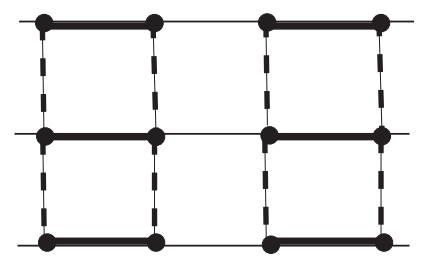

(b)
FIG. 8. Dimerized three-leg ladder. Bold, thin, and dashed lines represent the stronger or weaker chain coupling $J(1 \pm \delta)$ and rung coupling $J_{\perp}$, respectively. Dimerization patterns: (a) staggered; (b) columnar. valence bond solid (VBS) states. A particular VBS state is denoted according to the number of valence bonds formed with contiguous states; i.e., $(m, n)$-VBS with $m+n=3 S$. A transition from a $(1,2)-(\mathrm{VBS})$ phase to a $(2,1)-\mathrm{VBS}$ phase is understood to occur on crossing the phase boundary. ${ }^{11}$ The finite-size data for the energy gap was used to extract the critical indices near the transition point. This was done using a critical scaling analysis similar to that used to study the two-legged case, with the critical scaling relation given by Eq. (4). Data for ladders with $N=12$ to 30 were used. The scaling exponents on each side of the critical point, denoted as region $(1,2)(g<0)$ and region $(2,1)(g>0)$, were found to be different, with separate values $v_{(1,2)}$ and $v_{(2,1)}$. Collapse of the scaling function for $\delta=0.5$ (see Figs. 11 and 12) yields $v_{(1,2)} \approx 0.863, v_{(2,1)} \approx 0.385$ and $J_{\perp c} \approx 1.08$. For $\delta=1$, the

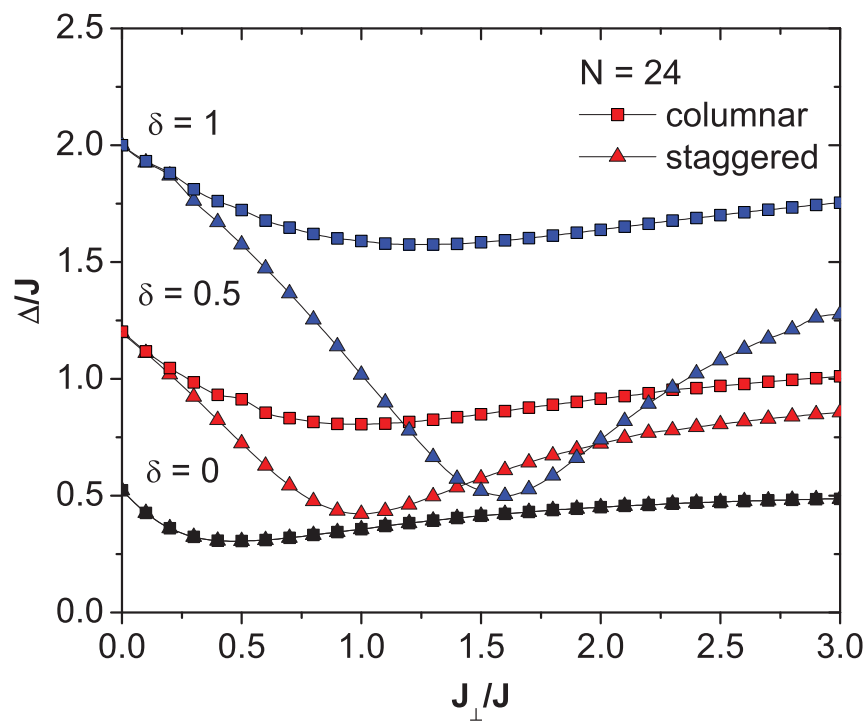

FIG. 9. (Color online) Energy gaps for the staggered and columnar three-leg ladder dimerization patterns calculated by the exact diagonalization for $N=24$. 


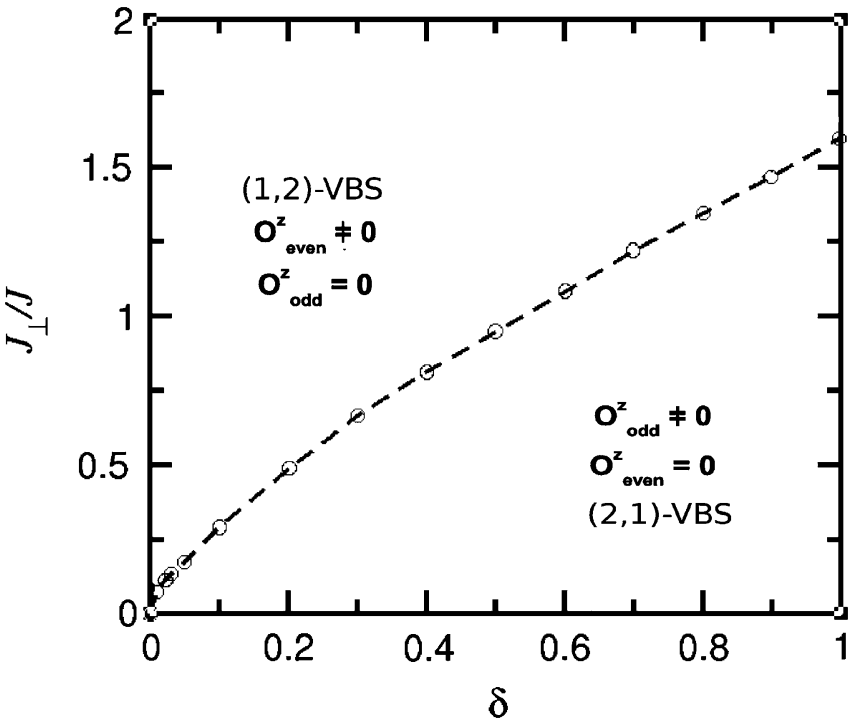

FIG. 10. Three-leg ladder; critical line $J_{\perp c}(\delta)$ where the gap of the staggered phase vanishes. Adapted from Ref. 12, original data from Ref. 11.

fitting gives $v_{(1,2)} \approx 0.789, v_{(2,1)} \approx 0.363$, and $J_{\perp c} \approx 1.65$. As opposed to the known behavior for large values of rung coupling $J_{\perp}$ of the gap of the two-legged ladder, which increases as $\Delta \propto J_{\perp}$, the gap of the three-legged ladder quickly saturates in both dimerization patterns (see Fig. 13). This fact limits the width of the critical region where the finite-size scaling analysis is valid.

\section{B. String-order parameter}

The generalized SOP for three-leg ladders is given by Eq. (5), where $S_{\text {rung }}^{z}(n) \equiv S_{1}^{z}(n)+S_{2}^{z}(n)+S_{3}^{z}(n)$ and $S_{\text {diag }}^{z}(n) \equiv S_{1}^{z}(n)+S_{2}^{z}(n+1)+S_{3}^{z}(n+2)$. The SOPs were calculated for $N=24$ for both the staggered and columnar configurations. Results for smaller systems $(N=12-18)$ give unreliable results due to the very small number of rungs in the sum. For this reason, a critical scaling analysis cannot

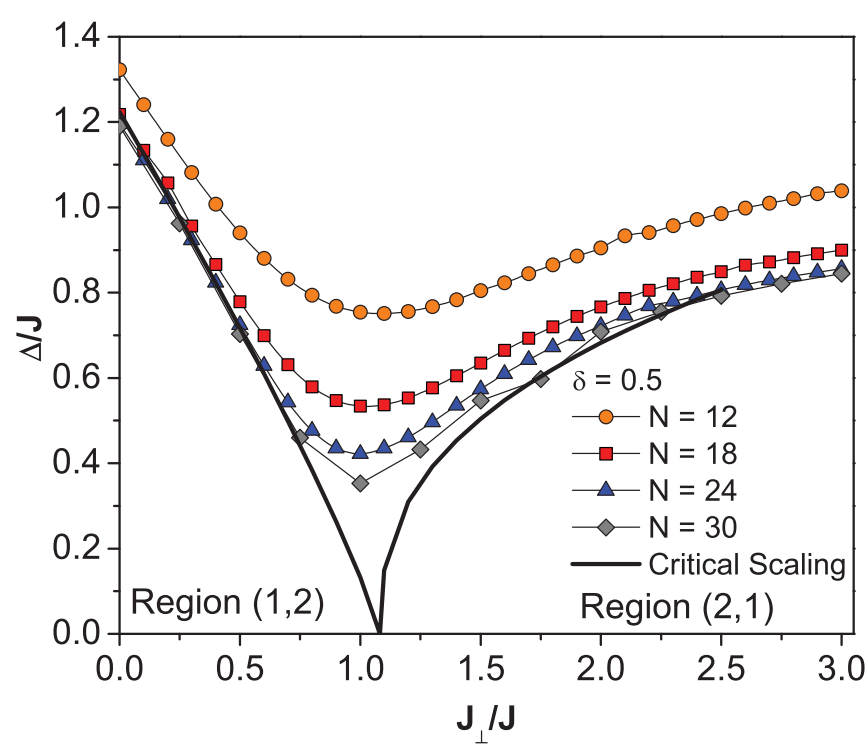

FIG. 12. (Color online) The collapsed scaling functions for the staggered three-leg ladder with $\delta=0.5$ in region $(1,2)$ (left-hand panel) and region $(2,1)$ (right-hand panel), calculated for $N=12$ through 30 with optimized parameters $v_{(1,2)} \approx 0.863, v_{(2,1)} \approx 0.385$, and $J_{\perp c} \approx 1.08$.

be performed for an estimate of $\beta$ until much larger system sizes $(N=30-36)$ are reached. Similar to the two-legged case, the columnar pattern was found to possess even string order throughout the parameter space, with no critical features. The staggered phase of the ladder possesses even string order associated with the $(1,2)$-VBS phase for $0<\delta<\delta_{c}$, which vanishes upon entering the $(2,1)$-VBS phase, accompanied by emerging odd string order. Representative results for the SOP in the staggered ladder are given in Fig. 14. However, we note that a residual odd string order persists in the $(1,2)$-VBS region of the parameter space. We believe this effect is due to the very small size of the system and should disappear if larger system sizes are assessed.
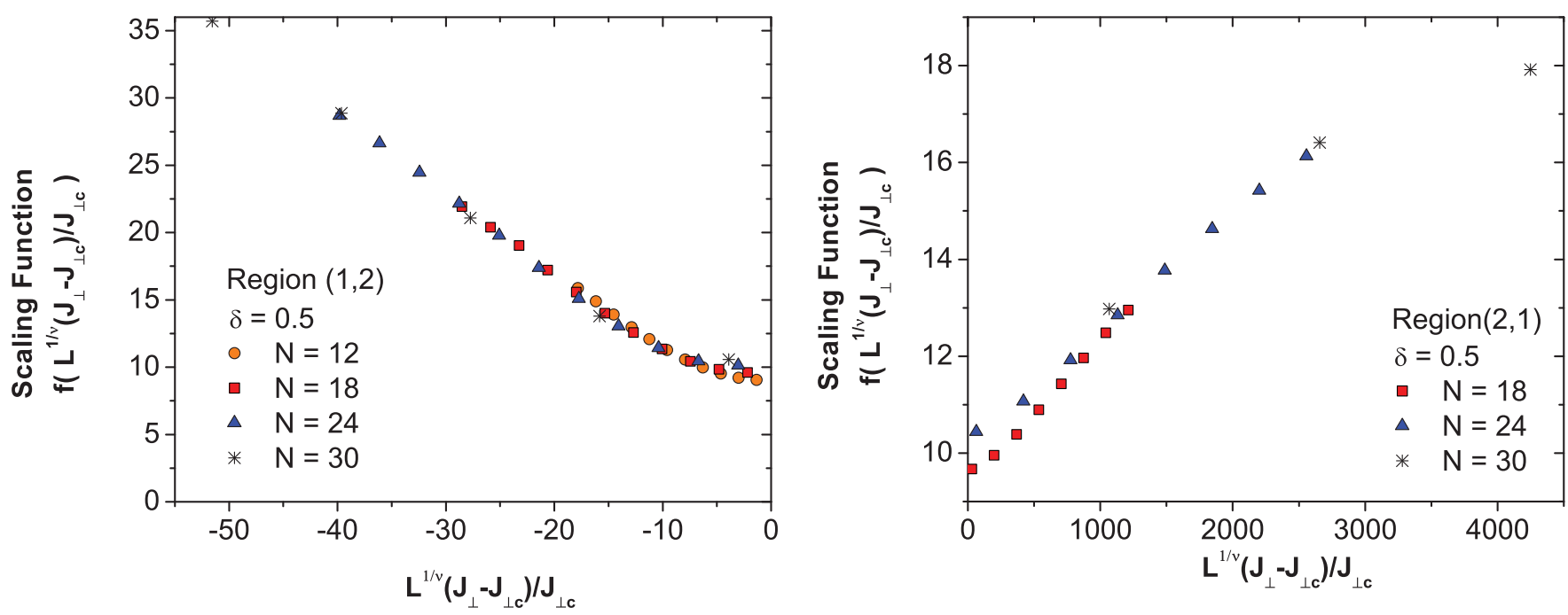

FIG. 11. (Color online) Three-leg ladder; gap saturation for strong rung couplings $J_{\perp}$. 


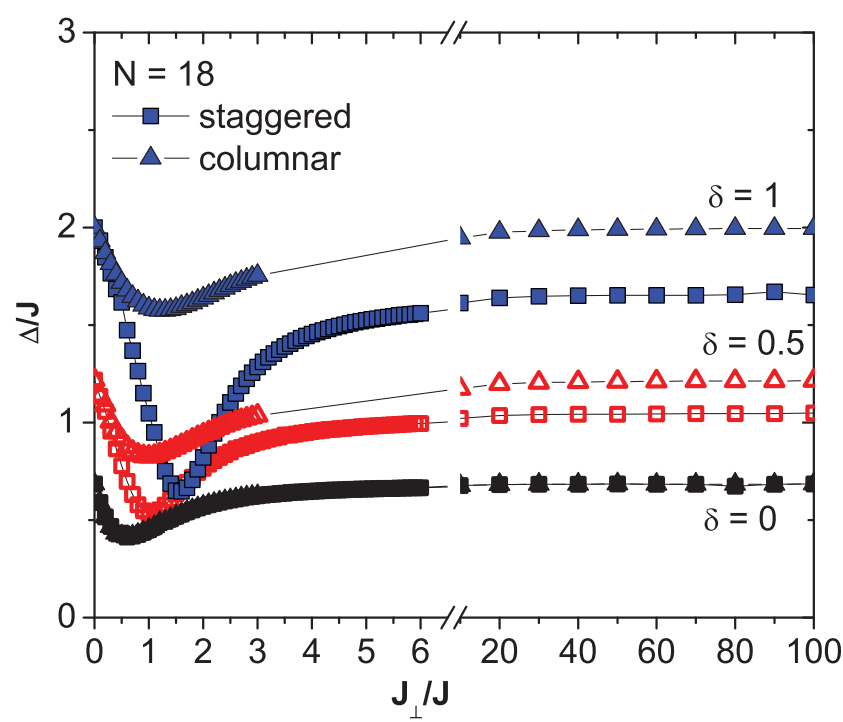

FIG. 13. (Color online) The ED data for the ground-state energy gap with $\delta=0.5$ for number of spins $N=12$ to 30 , along with the fit using optimized parameters $v_{(1,2)}=0.863$ in region $(1,2), v_{(2,1)}=$ 0.385 in region $(2,1)$, and $J_{\perp c}=1.08$.

\section{CONCLUSIONS}

We analyze the dimerized antiferromagnetic spin- $\frac{1}{2}$ twoand three-leg ladders. In agreement with the earlier meanfield predictions, ${ }^{12}$ the present ED results demonstrate that the columnar phases have lower ground-state energies than the staggered phases. Thus, if no mechanism for locking into a particular dimerization pattern is provided, a dimerized ladder would order in the columnar phase. In this paper we analyze the two possible dimerization patterns on the same footing, as intrinsic given properties of the ladders without providing specific mechanisms responsible for a particular dimerization.

The existence or absence of the quantum critical point is dependent on the dimerization pattern. The gapped phases, which cannot be distinguished by the conventional Landau long-range order parameter, have been characterized using the nonlocal SOPs, which are nonzero in one phase and vanish in the other. Here, for the first time, we systematically identify and numerically calculate all SOPs for the both critical (staggered) and noncritical (columnar) phases in the two-dimensional space of the model's couplings $\left(\delta, J_{\perp} / J\right)$. In addition we analyze the critical parameters near quantum criticality.

For both two- and three-leg ladders the columnar pattern was found to be always gapped and to possess the nonzero even SOP, which is essentially featureless. The finite-size ED data suggest that the odd SOP vanishes in the limit $N \rightarrow \infty$ in the columnar phase for both types of ladders considered.

The finite-size data for the energy gap were used to extract the critical indices in the vicinity of the line of quantum phase transition in the staggered case. The scaling behavior of the three-leg ladder was found to be different on each side of the critical point. These results give for $\delta=0.5$ values of $v_{(1,2)} \approx 0.863$ and $v_{(2,1)} \approx 0.385$.

From the critical scaling analysis of the ED data for the ladder with two legs we find the critical indices of the

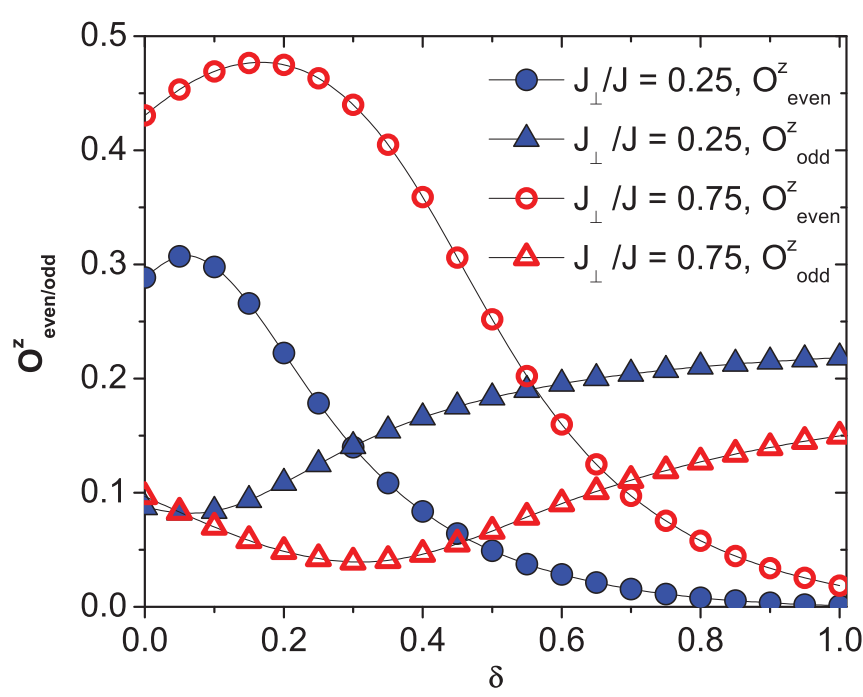

FIG. 14. (Color online) The odd and even string-order parameters of the staggered two-leg ladder with $J_{\perp} / J=0.25$ and 0.75 as functions of dimerization $\delta$. The ED data for ladders with $N=24$ total number of spins is shown.

correlation length $v \approx 0.755$, of the odd order parameter $\beta_{\text {odd }} \approx 0.2$ and of the even order parameter of $\beta_{\text {even }} \approx 0.02$. The rest of the critical indices can be determined by the scaling relations.

Most likely, the smallness of $\beta_{\text {even }}$ and large difference between $\beta_{\text {even }}$ and $\beta_{\text {odd }}$ are due to stronger finite-size artifacts in $O_{\text {even }}^{z}$. The nonmonotonous behavior of $O_{\text {even }}^{z}$, clearly seen in Fig. 6, also makes the finite-size scaling analysis less reliable.

According to the predictions of Refs. 4,5 and 8 the critical behavior of the two-leg ladder along the critical line $J_{\perp c}(\delta)$ is the same as near the integrable point $\left(J_{\perp} / J=2, \delta=1\right){ }^{27}$ Since along the line $\delta=1$ the ladder (1) reduces to a dimerized Heisenberg chain, the above assumption implies a critical behavior with $v=2 / 3$ and $\beta=1 / 12$ modified by multiplicative logarithmic corrections due to the marginally irrelevant term in the effective Hamiltonian. Our values $v \approx 0.75$ and $\beta \approx 0.2$ (including the case $\delta=1$ ) are comparable with these predictions, especially taking into account (i) natural limitations on the precision for the critical indices due to the small system sizes, which can be reached by ED, and (ii) fitting the critical indices without logarithmic corrections, which exaggerates their values (see, e.g., Refs. 28,29). The critical behavior of the ladders in the vicinity of (or along) the critical lines clearly warrants a detailed analytical study. We plan to address this issue in our future work.

\section{ACKNOWLEDGMENTS}

The authors thank E. H. Kim for fruitful discussions. SJG would also like to thank C. Gros, H. Merz, and A. Langille. Support from NSERC (Canada), Laurentian University Research Fund (LURF), Laurentian University, and the Shared Hierarchical Academic Research Computing Network (SHARCNET) is gratefully acknowledged. 
${ }^{1}$ E. Dagotto and T. M. Rice, Science 271, 618 (1996).

${ }^{2}$ T. Giamarchi, Quantum Physics in One Dimension (Oxford University Press, Oxford, 2004).

${ }^{3}$ L. D. Landau and E. M. Lifshitz, Statistical Physics Part 1, Course of Theoretical Physics, Vol. 5, 3rd ed. (Butterworth-Heinemann, London, 1980).

${ }^{4}$ M. A. Martin-Delgado, R. Shankar, and G. Sierra, Phys. Rev. Lett. 77, 3443 (1996).

${ }^{5}$ M. A. Martin-Delgado, J. Dukelsky, and G. Sierra, Phys. Lett. A 250, 430 (1998).

${ }^{6}$ V. N. Kotov, J. Oitmaa, and Z. Weihong, Phys. Rev. B 59, 11377 (1999).

${ }^{7}$ D. C. Cabra and M. D. Grynberg, Phys. Rev. Lett. 82, 1768 (1999).

${ }^{8}$ Y.-J. Wang and A. A. Nersesyan, Nucl. Phys. B 583, 671 (2000).

${ }^{9}$ K. Okamoto, Phys. Rev. B 67, 212408 (2003).

${ }^{10}$ M. Nakamura, T. Yamamoto, and K. Ide, J. Phys. Soc. Jpn. 72, 1022 (2003).

${ }^{11}$ J. Almeida, M. A. Martin-Delgado, and G. Sierra, Phys. Rev. B 76, 184428 (2007); 77, 094415 (2008); J. Phys. A 41, 485301 (2008).

${ }^{12}$ G. Y. Chitov, B. W. Ramakko, and M. Azzouz, Phys. Rev. B 77, 224433 (2008).

${ }^{13}$ C. Lanczos, J. Res. Natl. Bur. Stand. 45, 255 (1950).

${ }^{14}$ M. Azzouz, K. Shahin, and G. Y. Chitov, Phys. Rev. B 76, 132410 (2007).
${ }^{15}$ M. Azzouz, Phys. Rev. B 68, 174523 (2003).

${ }^{16}$ J. Cardy, Scaling and Renormalization in Statistical Physics (Cambridge University Press, Cambridge, 1996).

${ }^{17}$ M. den Nijs and K. Rommelse, Phys. Rev. B 40, 4709 (1989).

${ }^{18}$ M. Oshikawa, J. Phys. Condens. Matter 4, 7469 (1992).

${ }^{19}$ Y. Nishiyama, N. Hatano, and M. Suzuki, J. Phys. Soc. Jpn. 64, 1967 (1995).

${ }^{20}$ D. G. Shelton, A. A. Nersesyan, and A. M. Tsvelik, Phys. Rev. B 53, 8521 (1996).

${ }^{21}$ E. H. Kim, G. Fath, J. Solyom, and D. J. Scalapino, Phys. Rev. B 62, 14965 (2000).

${ }^{22}$ E. H. Kim, O. Legeza, and J. Solyom, Phys. Rev. B 77, 205121 (2008).

${ }^{23}$ G. Fath, O. Legeza, and J. Solyom, Phys. Rev. B 63, 134403 (2001).

${ }^{24}$ S. R. White, Phys. Rev. B 53, 52 (1996).

${ }^{25}$ H. Watanabe, Phys. Rev. B 52, 12508 (1995).

${ }^{26}$ K. Hida, Phys. Rev. B 45, 2207 (1992).

${ }^{27}$ We thank E. H. Kim for clarifying this point to us and bringing pertinent references to our attention.

${ }^{28}$ K. Okamoto, H. Nishimori, and Y. Taguchi, J. Phys. Soc. Jpn. 55, 1458 (1986).

${ }^{29}$ T. Papenbrock, T. Barnes, D. J. Dean, M. V. Stoitsov, and M. R. Strayer, Phys. Rev. B 68, 024416 (2003); M. Kumar, S. Ramasesha, D. Sen, and Z. G. Soos, ibid. 75, 052404 (2007). 